\title{
Innovative methods of strengthening reinforced concrete structures
}

\author{
Dmitry Mailyan ${ }^{1}$, and Darya Bogomazyuk ${ }^{1, *}$ \\ ${ }^{1}$ Don State Technical University, Gagarin square, 1, Rostov-on-Don, Russia
}

\begin{abstract}
The article considers some of the most innovative methods of reinforced concrete compressed elements: strengthening of elements with the help of pre-compressed longitudinal reinforcing elements, strengthening by means of concrete padding device, of low strength concrete, to strengthen the elements by biaxial transverse compression of concrete and method is to strengthen the elements with a pre-stretched diagonal reinforcement. The test results on deformation and rigidity of compressed reinforced concrete pillars are given. For each variant of rack reinforcement, depending on their flexibility and the relative eccentricity of the external force, the most technologically and economically feasible method of amplification is proposed.
\end{abstract}

\section{Introduction}

During the reconstruction of residential blocks in urban developments, it is often required to inspect and strengthen the constructions of buildings and structures. This can be caused either by design errors, changed operating conditions, by the occurrence of unforeseen situations because of the soaking with technogenic waters, violations of blind area integrity, rising groundwaters, etc.). All of the above causes can lead to defects in building structures that reduce their performance. Because of this it is of interest to improve the methods for restoring the operational usefulness of building structures, using both traditional (metal and concrete) and new, for example, composite materials.

\section{Research objectives and tasks}

The purpose of the study is to choose the most technologically and economically feasible way to strengthen concrete elements depending on their flexibility and relative eccentricity of external force.

\section{Description of the methods of reinforcement of structures}

Let's consider some of the most innovative methods for reinforcing reinforced concrete compressed elements, developed with the participation of the authors.

\footnotetext{
* Corresponding author: gor.d@list.ru
} 
The first method is to strengthen the elements with a precompressed longitudinal reinforcement [1].

The basic idea is to create a preliminary compression of the rods 4 of high-strength steel (Figure 1). The latter with steel plates 6 are attached to the main working armature of the columns.

The opposite ends of the rods 4 are passed through the steel corner supports 7 , also welded to the longitudinal reinforcement of the columns. To ensure the stability of the individual rods 4 , when they are pre-compressed, they are combined into a space frame by means of closed clamps 5, to the corners of which the rods 4 are fastened with a knitting wire. Preliminary compression of rods 4 is carried out by screwing nuts 8 . The control of the compressive force in them is made by measuring deformations.

a)

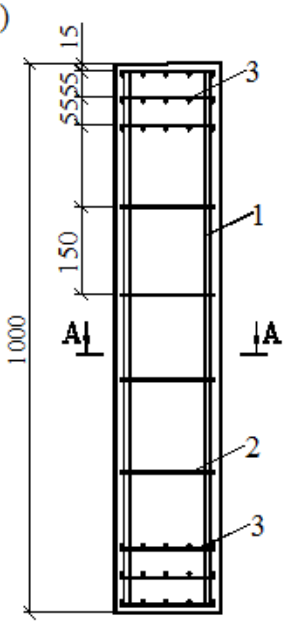

b)

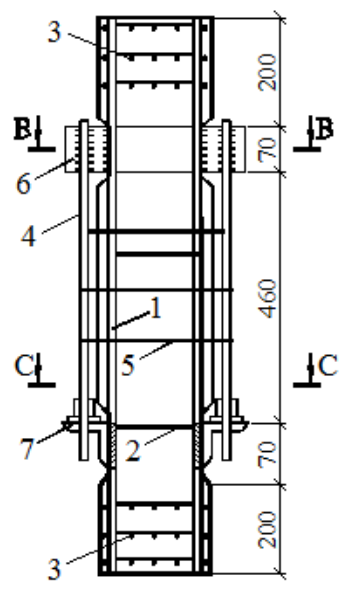

c)

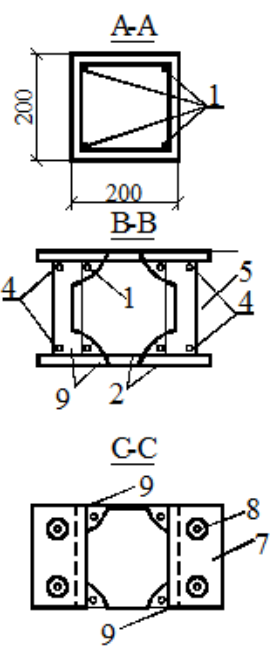

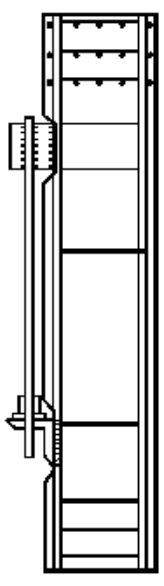

Fig. 1. Columns reinforced with pre-compressed elements: a - control non-amplified; b symmetrically reinforced; $\mathrm{c}$ - unilaterally strengthened; 1 and 2 - longitudinal and transverse reinforcement of the column; 3 - grids; 4 - pre-compressible reinforment elements; 5 - closed clamps; 6 - steel plates; 7 - corners (stops); 8 - nuts; 9 - welding.

$$
\begin{gathered}
P_{\mathrm{c}, \mathrm{con}}=P_{\mathrm{c}}-a_{\mathrm{sc}}\left(P_{\mathrm{c}} / A_{\mathrm{red}}\right) A_{\mathrm{st}} \\
P_{\mathrm{c}}=a_{\mathrm{s}} A_{\mathrm{st}} \sigma_{\mathrm{spc}} \\
A_{\text {red }}=a_{\mathrm{s}} A_{\mathrm{s}}+a_{\mathrm{sc}} A_{\mathrm{st}}
\end{gathered}
$$

$\sigma_{\mathrm{spc}}-$ pre-compression value of rods 4 ;

$A_{\mathrm{s}}$ and $A_{\mathrm{st}}$ - the cross-sectional area of the longitudinal reinforcement 1 and the reinforcement elements, respectively 4 ;

$a_{\mathrm{s}}$ and $a_{\mathrm{sc}}$ - reduction factors for reinforcements $A_{\mathrm{s}}$ and $A_{\mathrm{st}}$.

The strain state caused by the preliminary compression of the rods 4 (before the elastic strain is extinguished by an external load):

in the armature $A_{\mathrm{st}}-\sigma_{\mathrm{c}, \mathrm{con}}$

in fittings $A_{\mathrm{s}}$ - stretching $\sigma_{\mathrm{s}}=a_{\mathrm{s}} \sigma_{\mathrm{bt}}$. 
After extinguishing the elastic strains of concrete stretching by an external compressive strain in the armature $A_{\text {st }}$ and in concrete - zero.

When the strength of the reinforced section of the column is exhausted, the breaking force

$$
N=R_{\mathrm{b}} A_{\mathrm{b}}+R_{\mathrm{sc}} A_{\mathrm{s}}+\left(R_{\mathrm{sc}}+\sigma_{\mathrm{spc}}\right) A_{\mathrm{st}}
$$

Increase the strength of the column by installing compressed rods 4 , can be quite significant. For example, $R_{\mathrm{sc}}$ for primary reinforcement 1 does not exceed $400 \mathrm{MPa}$, and for reinforcement bars 4 of steel of class A1000 with its precompression $\sigma_{\mathrm{spc}}=400 \mathrm{MPathe}$ total stress at the destruction of columns reaches $800 \mathrm{MPa}$. Thus, if the rods 1 and 4 take the same diameter, then the force perceived by the rods of the original column will be 400 As, strengthened rods - $1200 \mathrm{As}$, as seen it will increase 3 fold.

It is often advisable to close the reinforcement structures by means of concrete of low strength. This protects the structure of the reinforcement with the help of concrete of low strength. This protects the reinforcement structures from corrosion. The strength of the reinforced section of the column is:

$$
N=R_{\mathrm{b}} A_{\mathrm{b}}+R_{\mathrm{bH}} \Delta A_{\mathrm{b}}+R_{\mathrm{sc}} A_{\mathrm{s}}+\left(R_{\mathrm{sc}}+\sigma_{\mathrm{spc}}\right) A_{\mathrm{st}}
$$

Cross-sectional area of concrete $\Delta A_{\mathrm{b}}$ arround $8 \mathrm{~cm}$ for columns with a cross-section of $40 \mathrm{x} 40 \mathrm{~cm}$ is arround $40 \%$ of the cross-section of the column, the strength of concrete $R_{\mathrm{bH}}$ is practicaly 3-4 times below the base value $R_{\mathrm{b}}$. Hence it is clear that the reinforcement increases the load-bearing capacity of the concrete section by $10 \ldots 15 \%$.

In columns of $40 \times 40 \mathrm{~cm}$ section from concrete of class $\mathrm{B} 30$ with reinforcement $4 \emptyset 20 \mathrm{~A} 600$ with steel reinforcement $4 \varnothing 20 \mathrm{~A} 1000$ and $\sigma_{\mathrm{spc}}=400 \mathrm{MPa}$ the strength of the reinforced area increases by 1.4 times. If the strength of the initial non-reinforced column is $2980 \mathrm{kN}$, then after amplification $-4235 \mathrm{kN}$, more than $1000 \mathrm{kN}$ are accounted for by the steel elements of amplification.

None of the known methods lead to such a significant effect as the one under consideration.

In columns operating with one-sided eccentricity of the resultant external longitudinal forces (racks of overpasses, overpasses, bridges, etc.), as well as when columns are damaged only on one side, reinforcement of the columns may be required only at the face closest to the resultant external forces or located with the damaged side of the column. In these cases, the precompressed elements 4 can be installed only about one face (Fig. 1, c). This will cause the center of gravity to shift in the reinforcement zone relative to the axis of the column. With eccentric compression, the axial eccentricity of the longitudinal force decreases.

In some cases, for example, in eccentrically compressed columns, it may be expedient to create an uneven preliminary stretching of the reinforcement section of the column. This can be achieved by unequal precompression of the reinforcing elements 4 located at two opposite faces. $[2]$.

Another way is to strengthen the elements by biaxial transverse compression of concrete

The creation of a biaxial compression of concrete in a direction perpendicular to the line of action of the external longitudinal compressive force increases the resistance of the element to transverse expansion. This leads to a "clip effect" and an increase in the bearing capacity of the element.

In the area to be strengthened, with a certain step in both directions, thrusts are set up (Figure 2), having at one end a seeded head or a nut. At the other end, a sleeve 6 and a nut 7 
are provided, which, when screwed, creates a tensile force in the rod. The reactive compressive force through the steel plates 4 is transferred to the body of the column.

a)

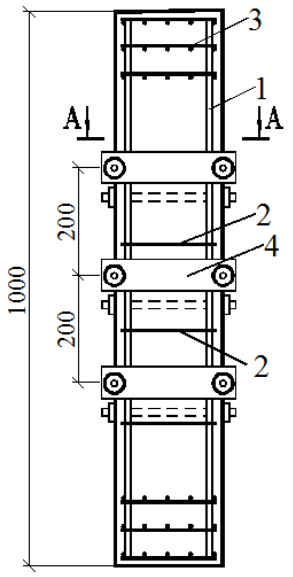

b)

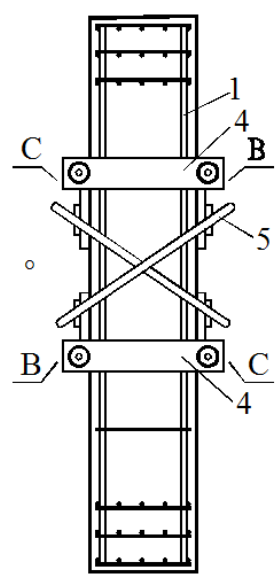

$\underline{\mathrm{A}-\mathrm{A}}$
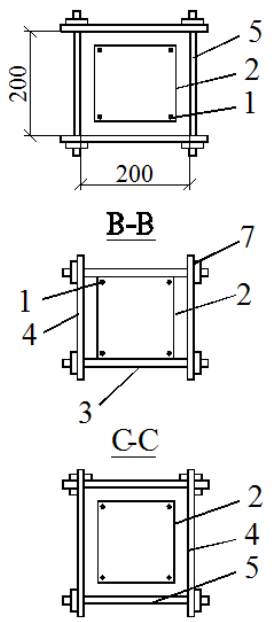

c)

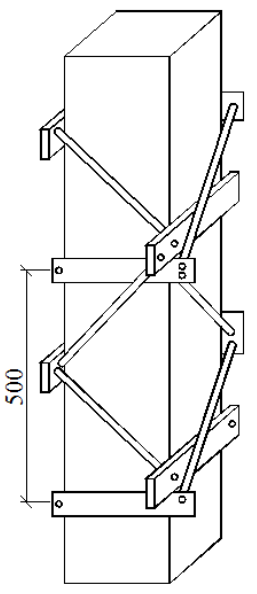

Fig. 2. Column reinforcement with pre-stretched elements a - with biaxial pre-compression of concrete; $b$ - with triaxial; in the same way, with drafts in different levels 1 - longitudinal reinforcement; 2 - transverse; 3 - grids; 4 - steel plates; 5 - traction; 6 - coupling; 7 - nut.

The preliminary compression in each of the two transverse directions (Figure 2) will be

$$
\sigma_{b p w}=\frac{2 \sigma_{s p} A_{s t}}{b s_{n}+a A_{s w} s_{n} / S}
$$

$\sigma_{\mathrm{spc}}$ - prestressing in rods;

$A_{\text {st }}$ - cross-sectional area of one rod;

$b$ - side cross-sectional dimension of a column perpendicular to stress direction $\sigma_{\mathrm{sp}}$;

$S_{n}$ - step of transverse reinforcement of the column in the area of amplification;

$A_{\text {sw }}$ - the cross-sectional area of the column reinforcement in one normal to the axis element of the plane; $a=\mathrm{E}_{\mathrm{s}} / \mathrm{E}_{\mathrm{b}}$.

Let us estimate the numerical values of these strain in the experimental elements. Let us consider rods with a diameter of $12 \mathrm{~mm}\left(A_{\mathrm{st}}=1,131 \mathrm{~cm}^{2}\right)$, transverse columns of $5 \mathrm{~mm}$ in diameter, column cross-section $20 \times 20 \mathrm{~cm}, \mathrm{~S}=\mathrm{S}_{\mathrm{n}}=20 \mathrm{~cm}, \mathrm{a}=6$ (concrete class B30, transverse reinforcement class A400). The force of preliminary stretching in one rod will be equal to $40 \mathrm{kN}$, then $\sigma_{\mathrm{sp}}=4000 / 1,131=353,7 \mathrm{MPa}$, and transverse reduction of concrete

$\sigma_{\text {spw }}=(2 \cdot 4000) /(20 \cdot 10+6 \cdot 0,393)=2 \mathrm{MPa}$

At this level of transverse reduction of concrete, significant changes in the diagram $« \sigma_{\mathrm{sp}}$ $-\varepsilon_{\mathrm{b}} \gg$. The extreme point shifts up and to the right. This significantly increases the loadbearing capacity of reinforced concrete columns.

The next method is to strengthen the elements with a pre-stretched diagonal reinforcement [3].

The reinforcement of the columns is carried out by preliminary stretching of the diagonal links 5 compressive forces on the concrete and longitudinal reinforcement of the 
columns through the steel plates 4 and fork stops 6 (Figure 2). The links are arranged in pairs on all four faces. Rods at one end are equipped with a seeded head or nut 7 with washers, and on the other - with couplings 6 and nuts 7. By twisting the latter, a tensile force is created in the rods. To fix the position of the rods to the longitudinal armature, fork stops (stoppers) are welded, differing only in height, which is caused by the necessity of locating the rods of the two directions at different distances from the faces of the columns.

When the diagonal reinforcement is stretched, the concrete is compressed in three mutually perpendicular directions (Figure 2, b), and the longitudinal and transverse reinforcement is compressed along its axes.

The strain state of the element after the preliminary stretching of the diagonal reinforcement in the cross section normal to the longitudinal axis of the element is characterized as follows:

strain in concrete $-\sigma_{\mathrm{bpc}}$ (compression);

strain in the armature $A_{\mathrm{s}}-\sigma_{\mathrm{spc}}$ (compression);

strain in diagonal reinforcement $A_{\text {inc }}-\sigma_{\text {sp,inc }}$ (stretching).

The destructive force of the element under the action of an external compressive load

$$
N=R_{\mathrm{b}, \text { red }} A_{\mathrm{b}}+A_{\mathrm{s}}\left(R_{\mathrm{sc}, \text { red }}-\sigma_{\mathrm{spc}}\right)+A_{\mathrm{s}, \text { inc }}\left(R_{\mathrm{sc}, \text { red }}-\sigma_{\mathrm{sp}, \text { inc }}\right) \cos a
$$

$\mathrm{R}_{\mathrm{b} \text {,red }}$ - reduced prismatic strength of concrete, taking into account the effect of the cage caused by biaxial transverse compression of concrete and changing the properties of concrete subjected to triaxial precompression;

$\mathrm{R}_{\mathrm{sc} \text {,red }}$ - reduced design resistance of reinforcement to compression taking into account increased compressibility of concrete;

$\sigma_{\mathrm{sp}, \text { inc }}$ - value of preliminary stretching of diagonal reinforcement;

$a$ - angle of inclination of diagonal reinforcement;

$A_{\text {s,inc }}$ - the cross-sectional area of all diagonal rods (if they are cross-arranged on all four faces - the cross-sectional area of the 8 rods).

Note that when axial compression of an element by an external load, the total longitudinal deformation of concrete will increase to the limiting $\varepsilon_{\mathrm{bu}}$ to the moment of destruction, and the total transverse deformations $\varepsilon_{\text {bu non }}$ they decrease even with a slight preliminary reduction, they can even change the sign. As a result, the length of the diagonal reinforcement decreases (Fig. 2, b)

$$
\left(1-\sqrt{\varepsilon_{b u}^{2} \pm \varepsilon_{b, n o n}^{2}}\right)
$$

- about $0,2 \%$.

Therefore, the preliminary stresses in it practically does not change. Therefore, the preliminary stresses. Let's consider a numerical example. For the column considered in the analysis of the first method, we take diagonal rods of $10 \mathrm{~mm}$ in diameter from steel of class A1000. With $\sigma_{\text {sp,inc }}=400 \mathrm{MPa}$ the tensile force in each of the eight diagonal rods is $\mathrm{P}=$ $31.4 \mathrm{kN}$, and the vertical and the horizontal components at $\emptyset=45^{\circ}$ are equal to $\mathrm{P} x$ $\cos \emptyset=22.6 \mathrm{kN}$. The longitudinal compression of concrete is $\sigma_{\mathrm{bpc}}=8 \times 2260 / 1600=1,4 \mathrm{MPa}$, and longitudinal compression of the reinforcement As $-\sigma_{\mathrm{spc}}=a \sigma_{\mathrm{bpc}}=10 \times 1,4=14 \mathrm{MPa}$.

These data show that the second term of expression (7) in comparison with the original $\left(\mathrm{R}_{\mathrm{sc}} \mathrm{A}_{\mathrm{s}}\right)$ will decrease by only $4 \%$, and the third term will be zero. Thus, the increase in the bearing capacity of the reinforced column in comparison with the initial one is determined by the first term of expression (7) and depends on the ratio of the $R_{b, \text { red }} / R_{b}$.

The next solution is to strengthen the elements with a pre-stretched diagonal reinforcement located at different levels [4]. 
In order to simplify the design of the gain and reduce metal consumption, as well as labor, pre-stretched diagonal links can be located at different levels (Figure 2c). With such a solution, the level of the triaxial reduction will decrease, but for certain conditions it can be sufficient.

Obviously, a reduction in the use of the diagonal reinforcement (per unit length of the column) will primarily reduce the longitudinal pre-stress. Therefore, this method is effective in columns of low flexibility, in which no appreciable increase in the hardness of the amplification of a large zone along the length of the column is required.

\section{Conclusions}

Thus, for each variant of rack reinforcement, depending on their flexibility and the relative eccentricity of the external force, the most technologically and economically feasible method of amplification is proposed.

\section{References}

1. D. Mailyan, V. Aksenov, N. Aksenov, Advances in Intelligent Systems and Computing, 692, 536-543 (2018)

2. D.R. Mailyan, R.L. Mailyan, M.V. Osipov, Concrete and reinforced concrete, 2, 18 (2002)

3. D.R. Mailyan, R.L. Mailyan, V.K. Khuranov, Proceedings of Higher Educational Institutions. Construction, 5, 4-11 (2004)

4. M. Khishmakh, D.R. Mailyan, P.P. Polskoy, A.M. Blyagoz, New Technologies, 4, 147-152 (2012) 